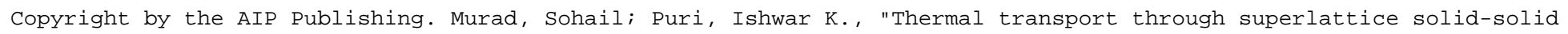

interfaces," Appl. Phys. Lett. 95, 051907 (2009); http://dx.doi.org/10.1063/1.3197012

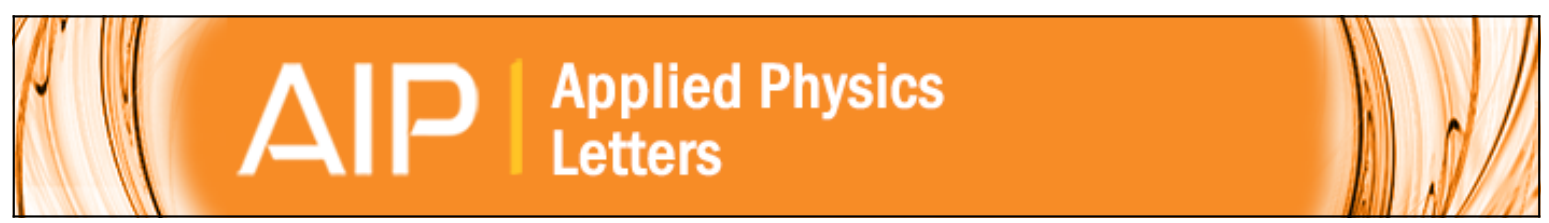

\title{
Thermal transport through superlattice solid-solid interfaces
}

Sohail Murad and Ishwar K. Puri

Citation: Applied Physics Letters 95, 051907 (2009); doi: 10.1063/1.3197012

View online: http://dx.doi.org/10.1063/1.3197012

View Table of Contents: http://scitation.aip.org/content/aip/journal/apl/95/5?ver=pdfcov

Published by the AIP Publishing

\section{Articles you may be interested in}

Few layer graphene based superlattices as efficient thermal insulators

Appl. Phys. Lett. 103, 141905 (2013); 10.1063/1.4824013

Heat conduction across a solid-solid interface: Understanding nanoscale interfacial effects on thermal resistance

Appl. Phys. Lett. 99, 013116 (2011); 10.1063/1.3607477

Interfacial thermal transport between nanotubes

J. Appl. Phys. 106, 084302 (2009); 10.1063/1.3245388

Unsteady nanoscale thermal transport across a solid-fluid interface

J. Appl. Phys. 104, 064306 (2008); 10.1063/1.2978245

Thermal transport across nanoscale solid-fluid interfaces

Appl. Phys. Lett. 92, 133105 (2008); 10.1063/1.2905281

\section{AlP Re-register for Table of Content Alerts}




\title{
Thermal transport through superlattice solid-solid interfaces
}

\author{
Sohail Murad ${ }^{1}$ and Ishwar K. Puri, ${ }^{2, a)}$ \\ ${ }^{1}$ Department of Chemical Engineering, University of Illinois at Chicago, Chicago, Illinois 60607, USA \\ ${ }^{2}$ Department of Engineering Science and Mechanics, Virginia Polytechnic Institute and State University, \\ Blacksburg, Virginia 24061, USA
}

(Received 1 July 2009; accepted 16 July 2009; published online 4 August 2009)

\begin{abstract}
Using molecular dynamics, we consider the thermal resistances of superlattices consisting of varying numbers of distinct nanolayers of two different materials. These are placed between two water reservoirs at uniform hot and cold temperatures. The interfacial resistances produced between different solid layers can lead to significantly lower heat transfer for a specified temperature difference. Such a large reduction in thermal transport cannot be explained by the interfacial resistance alone. In addition to the interfacial resistance between two adjacent superlattice layers, the relatively wide thermal boundary layers that are produced adjacent to the interfaces introduces a supplementary resistance. (C) 2009 American Institute of Physics. [DOI: 10.1063/1.3197012]
\end{abstract}

Phonon scattering at nanoscale interfaces ${ }^{1-4}$ produces a thermal contact resistance that impedes the heat flux. ${ }^{5,6}$ Thus, the nature of an interface has an important influence on thermal transport, ${ }^{7-10}$ e.g., through the formation of a thermal boundary layer adjacent to it. ${ }^{10}$ Combinations of thermal resistances in multilayered solid materials should decrease the heat transferred through their bulk as compared to, say, a homogeneous solid. Since the thermal resistance is inversely proportional to the interface temperature, ${ }^{11}$ the impedance to heat transfer should decrease with it. Interface effects cannot alone explain the reduction in the thermal conductivities ${ }^{12,13}$ of superlattices as compared to alloys with the same composition. ${ }^{14}$ We use molecular dynamics (MD) simulations to examine some unresolved issues by investigating model silicon-water like superlattice systems.

MD is applicable to phonon transport in nonmetallic solids above their Debye temperatures $T_{D} \cdot{ }^{14}$ We consider a system that consists of 4224 particles in the basic cyclically replicated parallelepiped that is described in Fig. 1. It consists of two solid $6.5 \mathrm{~nm}$ walls that separate $4 \mathrm{~nm}$ wide fluid reservoirs. ${ }^{8,10}$ Both walls consist of 3072 solid atoms in the form of $4 \times 4 \times 6$ unit cells that contain eight atoms. The fluid reservoirs contain 1152 uniformly distributed water molecules at a density of $975 \mathrm{~kg} / \mathrm{m}^{3}$. The solid atoms are of four siliconlike materials $M_{i}$, namely, $\mathrm{Si}$ (or $M_{1}$ ) or its close variants $M_{2}, M_{3}$, and $M_{4}$. These are placed at the normal equilibrium sites for a silicon crystal. We provide the molecules with initial Gaussian velocity distributions ${ }^{15}$ which impart different high and low temperatures, $T_{h} \geq T_{D}$ $\approx 645 \mathrm{~K}$ (for a nonmetallic $\mathrm{Si}$ crystal $^{16}$ ) and $T_{c}$ to the fluid reservoirs.

We simulate three solid-fluid systems with (1) homogenous walls consisting of a single material $M_{i}$, (2) composite walls comprised of a single $\mathrm{Si}-M_{i, i=2,3,4}$ bilayer, and (3) multilayer walls (superlattices) consisting of six Si- $M_{i, i=2,3,4}$ composite layers. Figure 1 schematically describes the latter two systems with $\mathrm{Si}-M_{4}$ layers. In all cases, the overall system length $L_{y}=21 \mathrm{~nm}$, and its height $L_{y}$ and width $L_{z}$ are both $2.172 \mathrm{~nm}$.

\footnotetext{
a) Author to whom correspondence should be addressed. Electronic mail: ikpuri@vt.edu.
}

We have employed the simulation algorithm, described elsewhere, ${ }^{15,17-19}$ to investigate various interfacial heat and mass transport problems. ${ }^{8-10,15,20-24}$ Intermolecular interactions follow the potential $u_{i j}=4 \varepsilon_{i j}\left[\left(\sigma_{i j} / r_{i j}\right)^{12}-\left(\sigma_{i j} / r_{i j}\right)^{6}\right]$ $+q_{i} q_{j} / r_{i j}$, where $\sigma_{i j}$ and $\varepsilon_{i j}$ denote the Lennard-Jones (LJ) interaction parameters, $r_{i j}$ the scalar distance between sites $i$ and $j$, and $q_{i}$ and $q_{j}$ charges on sites when applicable. The parameters $\sigma$ and $\varepsilon$, presented in Table I, represent experimental geometries, energies of ion-water complexes, and of solvation $^{25}$ for (1) water (modeled with the SPC potential) ${ }^{10,26}$ and (2) $\mathrm{Si}$ (or $M_{i}$ ) wall atoms modeled as LJ sites. $8,21,22$

We emulate the coordinated tetrahedral bonded Si structure by tethering the $M_{i}$ atoms to their equilibrium sites using a simple harmonic potential with a $61.7 \mathrm{~J} \mathrm{~m}^{2}$ spring constant. This leads to an ideal vibrational frequency for a simple harmonic oscillator of $19304 \mathrm{~cm}^{-1}$. Because the tethered molecules interact strongly with neighboring atoms, the actual vibrational frequency is lower than this value. The parameter values for the hypothetical $M_{i}$ solids are identical to those for $\mathrm{Si}$ except that $\sigma_{2}=1.067 \sigma_{\mathrm{Si}}, \sigma_{3}=1.1167 \sigma_{\mathrm{Si}}$, and $\sigma_{4}=1.167 \sigma_{\mathrm{Si}}$ for the interactions of $M_{1}, M_{2}$, and $M_{3}$ with other $M_{i}$ atoms. All $M_{i}$ atoms interact identically with water, since our purpose is to focus on the solid-solid resistances.

The simulated cases are summarized in Table II. Segmenting the simulation domain into $138 \Delta x \approx 0.152 \mathrm{~nm}$ thick transverse strips along $L_{x}$ provides averaged temperatures in

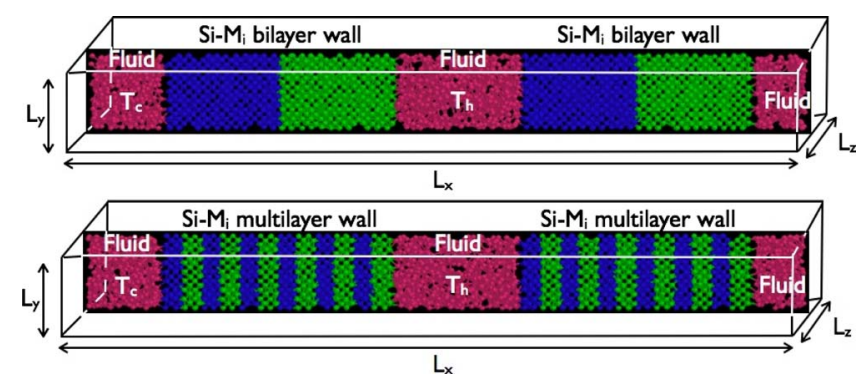

FIG. 1. (Color online) Schematic diagram of the domain based on an arbitrary simulation for systems with bilayer and six-layer composite Si ( or $M_{1}$ ) $-M_{i}$ superlattice walls. Periodic boundary conditions apply on all six faces of the basic cyclically replicated parallelepiped. The domain dimensions are $L_{x}=20.99 \mathrm{~nm}$ and $L_{y}=L_{z}=2.172 \mathrm{~nm}$. 
TABLE I. Values of the Lennard-Jones interactions parameters $\sigma, \varepsilon$, and $q$. Cross interactions are modeled based on Lorentz-Berthelot mixing rules.

\begin{tabular}{llll}
\hline \hline & $\begin{array}{c}\sigma \\
10^{-10} \mathrm{~m}\end{array}$ & $\begin{array}{c}\varepsilon \\
\mathrm{KJ} \mathrm{mol}^{-1}\end{array}$ & $\begin{array}{l}q \\
e\end{array}$ \\
\hline $\mathrm{O}$ & 3.17 & 0.65 & -0.82 \\
$\mathrm{H}$ & 0 & 0 & 0.41 \\
$\mathrm{Si}$ & 4.028 & 0.87 & 0 \\
\hline \hline
\end{tabular}

these local sections for each case. Figure 2 presents the temperature profiles for Cases I and Ia. These are converged within $2 \%$ of their reported local values over the last 2 $\times 10^{6}$ simulation steps. The temperature distribution for Case I reveals thermal boundary layers adjacent to the solid-fluid and solid-solid interfaces that form resistances which lower the effective thermal conductivity. ${ }^{10}$ The boundary layer thickness $\delta_{t} \approx 2 \Delta x$ or $0.3 \mathrm{~nm}$ is roughly a single atomic layer wide. Since it is identical in the $\mathrm{Si}$ and $M_{4}$ layers, the heat flux $Q$ through the composite wall is constant. Using the empirical Fourier law $Q=-k \nabla T$ where $k$ denotes the thermal conductivity, the lower effective $k$ for the $\mathrm{Si}$ wall is apparent through the steeper $\nabla T$ profile as compared to that for $M_{4}$. Placing thermal resistances in series in a composite six-layer $\mathrm{Si}-M_{4}$ wall further distorts the profile, since phonon scattering now occurs at multiple $\mathrm{Si}-M_{4}$ interfaces, decreasing $Q$ and thus $k$, as illustrated in Table II.

For the LJ model, increasing $\sigma$ augments the intermolecular interactions between two neighboring $M_{i}$ atoms or sites, i.e., a decrease in the interparticle distance enhances thermal transport. The heat flux through monolayer walls of a single material increases as $Q \propto \sigma^{3.64}$, i.e., thermal transport scales roughly with the material atomic volumes. Thus, varying the thickness of a layer should not influence this effect. Table II compares the relative heat flux (normalized against Case III) $Q / Q_{\mathrm{III}}$ for each case as a function of the wall composition and overall temperature difference across it. A $\sim 17 \%$ increase in molecular size (for $M_{4}$ - Case $\mathrm{X}$ and over $\mathrm{Si}$-Case VII) leads to a $\sim 80 \%$ increase in $Q$ for a $645 \mathrm{~K}$ temperature difference across the wall. A six-layer $\mathrm{Si}-M_{4}$ wall conducts less heat than a corresponding bilayer composite wall. The heat transferred across a six-layer wall is $5 \%$
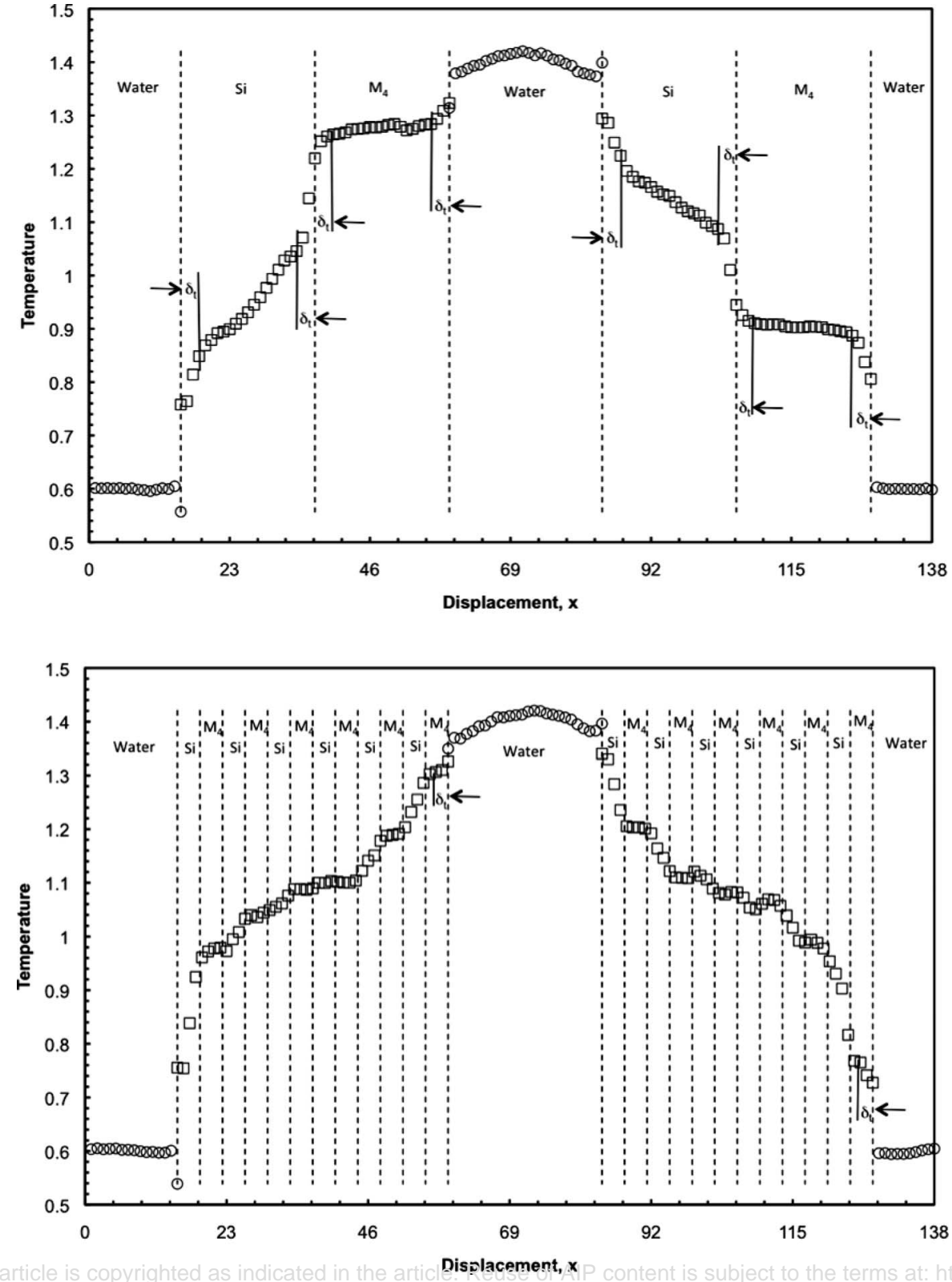

FIG. 2. The temperature profiles for Cases I and Ia. The segment numbers on the abscissa multiplied by 0.152 $\mathrm{nm}$ return the axial distance. The dimensionless temperature ordinate multiplied by $806.46 \mathrm{~K}$ provides the local temperature. 
TABLE II. List of simulation cases corresponding to the materials comprising the solid superlattice walls, numbers of composite $\left(M_{i}-M_{j}\right)$ layers in each wall, imposed hot- and cold-side fluid reservoir temperatures $T_{h}$ and $T_{c}$, simulated heat flux $Q$ through the superlattice walls, and the ratio of $Q$ to the flux for a base case (III). Cases VII-X are for homogeneous walls.

\begin{tabular}{lccccccc}
\hline \hline Case & $\begin{array}{c}\text { Material } \\
1\end{array}$ & $\begin{array}{c}\text { Material } \\
2\end{array}$ & $\begin{array}{c}\text { Composite } \\
\text { bilayers }\end{array}$ & $\begin{array}{c}T_{h} \\
(\mathrm{~K})\end{array}$ & $\begin{array}{c}T_{c} \\
(\mathrm{~K})\end{array}$ & $\begin{array}{c}Q \times 10^{7} \\
(\mathrm{~W})\end{array}$ & $Q / Q_{\mathrm{III}}$ \\
\hline I & $\mathrm{Si}\left(M_{1}\right)$ & $M_{4}$ & 1 & 1210 & 403 & 2.69 & 1.05 \\
Ia & $\mathrm{Si}\left(M_{1}\right)$ & $M_{4}$ & 6 & 1210 & 403 & 2.55 & 0.99 \\
II & $\mathrm{Si}\left(M_{1}\right)$ & $M_{4}$ & 1 & 1169 & 444 & 2.64 & 1.03 \\
IIa & $\mathrm{Si}\left(M_{1}\right)$ & $M_{4}$ & 6 & 1169 & 444 & 2.50 & 0.98 \\
III & $\mathrm{Si}\left(M_{1}\right)$ & $M_{4}$ & 1 & 1129 & 484 & 2.57 & 1.00 \\
IIIa & $\mathrm{Si}\left(M_{1}\right)$ & $M_{4}$ & 6 & 1129 & 484 & 2.19 & 0.85 \\
IV & $\mathrm{Si}\left(M_{1}\right)$ & $M_{4}$ & 1 & 1089 & 524 & 2.27 & 0.88 \\
IVa & $\mathrm{Si}\left(M_{1}\right)$ & $M_{4}$ & 6 & 1089 & 524 & 1.78 & 0.69 \\
V & $\mathrm{Si}\left(M_{1}\right)$ & $M_{4}$ & 1 & 1048 & 565 & 2.15 & 0.84 \\
Va & $\mathrm{Si}\left(M_{1}\right)$ & $M_{4}$ & 6 & 1048 & 565 & 1.28 & 0.50 \\
VI & $\mathrm{Si}\left(M_{1}\right)$ & $M_{2}$ & 1 & 1129 & 484 & 2.19 & 0.85 \\
VIa & $\mathrm{Si}\left(M_{1}\right)$ & $M_{2}$ & 6 & 1129 & 484 & 2.19 & 0.85 \\
VII & $\mathrm{Si}\left(M_{1}\right)$ & $\ldots$ & $\ldots$ & 1129 & 484 & 2.02 & 0.79 \\
VIII & $M_{2}$ & $\ldots$ & $\ldots$ & 1129 & 484 & 2.57 & 1.00 \\
IX & $M_{3}$ & $\ldots$ & $\ldots$ & 1129 & 484 & 2.86 & 1.11 \\
X & $M_{4}$ & $\ldots$ & $\ldots$ & 1129 & 484 & 3.63 & 1.41 \\
\hline \hline
\end{tabular}

smaller than the flux across a bilayer wall when the temperature difference across it is $806 \mathrm{~K}$ (Case Ia). For a $645 \mathrm{~K}$ difference the flux is reduced by $15 \%$ (Case IIIa) but its decrease is $41 \%$ at $484 \mathrm{~K}$ (Case Va). These changes in the thermal conductivity ${ }^{12,13}$ cannot be accounted for by the interface resistance alone. ${ }^{14}$ We attribute the decrease in $k$ also to the thermal boundary layers around the contact interface between two solid crystals. Lowering the temperature difference makes a multilayer wall less effective in transferring heat, since the thermal impedance is sensitive to the temperature difference and the interfacial temperature.

In summary, we show that impedance to nanoscale thermal transport is enhanced by subnanometer thermal boundary layers at the interfaces of superlattices due to two effects, namely, (1) the interfacial resistance between two solid phases of a superlattice and (2) the thermal boundary layers that are formed within each phase adjacent to these interfaces. This observation can have significant design implica- tions for semiconductor and related electronic devices that dissipate energy as heat. For example, depending upon the desired outcome, it would be advantageous to have composite films constructed in several alternating layers to inhibit heat transfer, but have fewer layers present if the thermal transport is to be enhanced.

This work was partially supported by a grant from the National Science Foundation (Grant No. CBET-0730026).

${ }^{1}$ L. J. Challis, J. Phys. C 7, 481 (1974).

${ }^{2}$ J.-L. Barrat and F. Chiaruttini, Mol. Phys. 101, 1605 (2003).

${ }^{3}$ C. Oligschleger and J. C. Schon, Phys. Rev. B 59, 4125 (1999).

${ }^{4}$ P. A. E. Schoen, B. Michel, A. Curioni, and D. Poulikakos, Chem. Phys. Lett. 476, 271 (2009)

${ }^{5}$ E. T. Swartz and R. O. Pohl, Rev. Mod. Phys. 61, 605 (1989).

${ }^{6}$ P. L. Kapitza, J. Phys. (USSR) 4, 181 (1941).

${ }^{7}$ G. Balasubramanian, S. Banerjee, and I. K. Puri, J. Appl. Phys. 104, 064306 (2008).

${ }^{8}$ S. Murad and I. K. Puri, Appl. Phys. Lett. 92, 133105 (2008).

${ }^{9}$ S. Murad and I. K. Puri, Chem. Phys. Lett. 467, 110 (2008).

${ }^{10}$ S. Murad and I. K. Puri, Chem. Phys. Lett. 476, 267 (2009).

${ }^{11}$ A. F. Khater, J. Phys. Colloq. 39, C6 (1978).

${ }^{12}$ S. M. Lee, D. G. Cahill, and R. Venkatasubramanian, Appl. Phys. Lett. 70, 2957 (1997).

${ }^{13}$ T. Borca-Tasciuc, W. L. Liu, J. L. Liu, T. F. Zeng, D. W. Song, C. D. Moore, G. Chen, K. L. Wang, M. S. Goorsky, T. Radetic, R. Gronsky, T. Koga, and M. S. Dresselhaus, Superlattices Microstruct. 28, 199 (2000).

${ }^{14}$ D. G. Cahill, W. K. Ford, K. E. Goodson, G. D. Mahan, A. Majumdar, H. J. Maris, R. Merlin, and S. R. Phillpot, J. Appl. Phys. 93, 793 (2003).

${ }^{15}$ M. P. Allen and D. J. Tildesley, Computer Simulation of Liquids (Clarendon, Oxford, 1987).

${ }^{16}$ H.-M. Kagaya, T. Soma, and M. R. Brozel, in Properties of Crystalline Silicon, edited by R. Hull (The Institution of Engineering and Technology, London, U.K., 1999), pp. 151-152.

${ }^{17}$ S. Murad and J. Lin, Chem. Eng. J. 74, 99 (1999).

${ }^{18}$ S. Murad and J. Lin, Ind. Eng. Chem. Res. 41, 1076 (2002).

${ }^{19}$ D. J. Evans and S. Murad, Mol. Phys. 34, 327 (1977).

${ }^{20}$ S. Banerjee, S. Murad, and I. K. Puri, Chem. Phys. Lett. 434, 292 (2007).

${ }^{21}$ S. Murad and I. K. Puri, Nano Lett. 7, 707 (2007).

${ }^{22}$ S. Murad and I. K. Puri, Phys. Fluids 19, 128102 (2007).

${ }^{23}$ I. G. Tironi, R. Sperb, P. E. Smith, and W. F. Vangunsteren, J. Chem. Phys. 102, 5451 (1995).

${ }^{24}$ H. J. C. Berendson, J. Postma, and W. F. van Gunsteren, in Intermolecular Forces, edited by B. Pullman (Reidel, Dordecht, 1981).

${ }^{25}$ J. Chandrasekhar, S. F. Smith, and W. L. Jorgensen, J. Am. Chem. Soc. 106, 3049 (1984)

${ }^{26}$ R. O. Watts, Mol. Phys. 28, 1069 (1974). 